\title{
Long Range Wireless Interrogation of Passive Humidity Sensors using Van-Atta Cross-Polarization Effect and 3D Beam Scanning Analysis
}

\author{
Dominique Henry ${ }^{1}$, Jimmy G.D.Hester ${ }^{2}$, Hervé Aubert ${ }^{1}$, Patrick Pons ${ }^{1}$ and Manos M. Tentzeris ${ }^{2}$ \\ ${ }^{1}$ LAAS-CNRS, Toulouse, 31031 France \\ ${ }^{2}$ School of Electrical and Computer Engineering, Georgia Institute of Technology, Atlanta, Ga 30332
}

\begin{abstract}
This paper reports for the first time a long-range interrogation ( $>50$ meters) of wireless and batteryless humidity sensors combining a Van-Atta retrodirective array and a 3D beam scanning using a 24GHz Frequency-Modulated Continuous-Wave radar. Van-Atta cross-polarization properties, as well as the use of dedicated statistical estimators and Synthetic Aperture Radar technique allow the long-range measurement of the relative humidity at a distance of 58 meters. A measurement sensitivity of $0.2 \mathrm{~dB}$ to $0.4 \mathrm{~dB}$ per $\% \mathrm{RH}$ was measured as a linear variation of the proposed estimator with a standard error of $\pm 0.005 \mathrm{~dB}$.

Index Terms - Chipless RFID, Flexible electronics, Internet of Things, Microstrip antenna arrays, antennas arrays, Radar imaging, Radar remote sensing.
\end{abstract}

\section{INTRODUCTION}

Nowadays measuring physical quantities wirelessly is no more a major issue with the explosive growth and utilization of active electronic devices with integrated sensors. These devices can be part of a network and, more generally, part of the Internet of Things (IoT). However it becomes challenging when physical quantities need to be measured in harsh environment. Human intervention must be limited in these abrupt conditions, such as very high or very low temperature and pressure, or even radioactivity. Hence wireless sensors with long-life duration and long achievable range are required. One possible solution is the use of remotely readable passive sensors (without integrated devices and DC power supply) because their life duration depends only on their constitutive material properties. However the main technical challenge lies in deriving the physical quantity of interest from the sensor electromagnetic echo for a very long interrogation range.

Several technologies are currently used to perform the remote interrogation of passive sensors. Radio-Frequency IDentification (RFID) technology is widely utilized in many applications for both tag identification (based on a barcode reading) and tag sensing. However, power regulations do not allow RFID to achieve a sufficient interrogation range (typically $<20$ meters) in applications where environments are harsh and highly reflective. Surface Acoustic Wave (SAW) sensors could also be a solution but for detection at ranges lower than 10 meters due to the high-loss electromagnetic-to-acoustic waves conversion.

In this paper we propose for the first time an alternative technique to the standard RFID interrogation in order to locate and remotely read flexible passive humidity sensors at long interro- gation ranges $(>50 \mathrm{~m})$. The proposed technique merges technologies from two different domains: (a) a Frequency-Modulated Continuous-Wave (FMCW) scanning method with signal processing, Synthetic Aperture Radar (SAR) and statistical estimators, (b) the design of passive sensors using humidity-sensitive Kapton-based Van-Atta retrodirective arrays. This technique was already investigated in [1] but was not reported using an integrated passive sensor and, at such long distance and measurement accuracy. Moreover, this kind of humidity passive sensors was wirelessly interrogated at only 5.5 meters [2] and detected at $30.0 \mathrm{~m}$ [3]. Such results require at least $4 \mathrm{GHz}$ of frequency band (13\%) around $30 \mathrm{GHz}$ to perform the fullscale dynamic range measurement.

The first part of the paper explains briefly the beam scanning technique as well as the properties of the Van-Atta reflect array. The second part is focused on the indoor interrogation of the humidity passive sensors at different distances from the radar reader $(1.3 \mathrm{~m}, 10.0 \mathrm{~m}$ and $58.0 \mathrm{~m})$. Different methods are investigated in order to reach the largest full-scale measurement range and the highest linearity by using a specific statistical analysis. A three-dimensional display of the echo levels using convenient isosurfaces is also reported with/without the presence of the proposed sensors in clutter environments. Remote reading of the Van-Atta reflect array at different angles of incidence is also discussed.

\section{WIRELESS INTERROGATION OF PASSIVE SENSORS}

\section{A. FMCW Radar Scanning Technique}

To perform the remote reading of sensors, a FMCW radar with a $23.8 \mathrm{GHz}$ central frequency is used here. This choice of carrier frequency brings many advantages such as a good achievable linearity of the Voltage Control Oscillator (VCO) in a wide frequency band $(\mathrm{B}=2 \mathrm{GHz})$, which is mandatory for reaching a sufficient depth resolution $d$ [4] $(d=c / 2 B=$ $7.5 \mathrm{~cm}$ where $c$ denotes the velocity of light), an operating frequency belonging to the ISM band and the design of small size passive sensors with integrated antennas.

A mechanical beam scanning is performed here. The transmitted (Tx) channel of the FMCW radar is connected to a rotating parabolic antenna with the gain of $33.5 \mathrm{dBi}$ and the beamwidth of $2^{\circ}$. The rotation has a step of $1^{\circ}$ in azimuth and elevation. The electromagnetic signals backscattered by the sensors are received by a $1 \times 5$ patch array antenna with the gain of 
8.6 $\mathrm{dBi}$ and the beamwidth of $60^{\circ}$ and $25^{\circ}$ in azimuth and elevation, respectively. Spatial translation of the radar system is also performed. Let $d f=2 D^{2} / \lambda$ be the Fraunhofer distancewhere $D=50 \mathrm{~cm}$ is the largest antenna dimension of the parabolic dish diameter. Consequently, the theoretical separation distance between far and near field regions stands at $d f=$ $39.6 \mathrm{~m}$. The radar output power is $20 \mathrm{dBm}$.

\section{B. Kapton-based Van-Atta passive humidity sensors}

The passive humidity sensor used for the measurements is a $74 \times 74 m^{2}$ Van-Atta reflectarray structure (see Fig 1 (a)). It is composed of five side-by-side linear antenna arrays and inkjetprinted on an easily low-cost and integrable flexible substrate (Kapton HN polymid). It has the property to retransmit in phase and cross-polarized incident waves. A description of its Radar Cross-Section (RCS) properties versus bending can be found in [2], [3]. Using the bistatic FMCW radar for linearly polarized incident electromagnetic fields facilitates the detection of the passive sensor by increasing the Signal-to-Noise Ratio (SNR). Moreover the sensor ground plane allows the proximity of metallic clutter without degrading its re-radiating properties.

The permittivity of the Kapton is very sensitive to humidity and a small change generates an observable shift of the device resonant frequency [5]. Thus an appropriately designed passive sensor at $24 \mathrm{GHz}$ provides a measurable echo level fluctuation. Wireless and indoor measurements of this sensor are performed in a long (60 meters) corridor (Fig. 1(b)). In comparison with an outdoor or a non-reflective environment, performing measurement indoors in such structure generates multipath and decreases the SNR. This is generally due to high reflective clutters such as metallic grids or walls. It is intended to perform these measurements in such conditions to validate the experiment in non-ideal conditions.

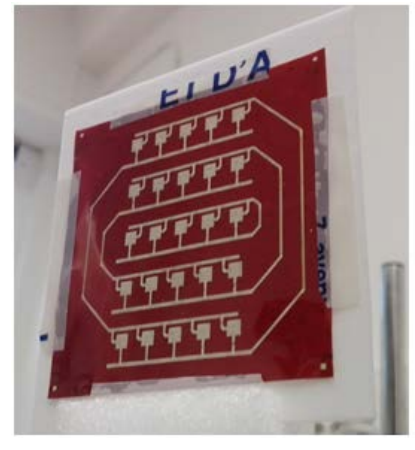

(a)

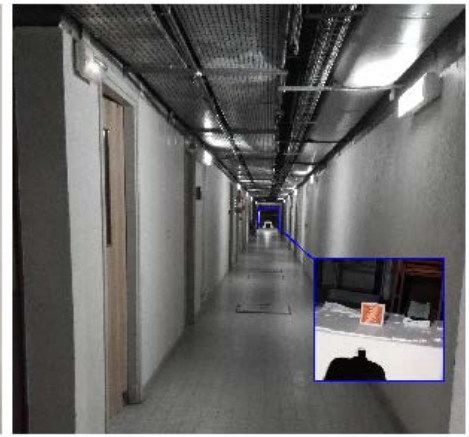

(b)
Fig. 1. (a) Kapton-based Van-Atta reflectarray prototype and (b) long range (60 meters) and indoor remote measurement setup of this structure in a corridor.

\section{MEASUREMENT RESULTS AND DISCUSSION}

\section{A. Indoor interrogation at $1.3 \mathrm{~m}$ and definition of statistical estimators}

Wireless measurements are first performed to study the linearity and the full-scale measurement range of the Van-Atta reflectarray. For this purpose the passive sensor is placed in a chamber with a humidity controller and a reference humidity sensor with an accuracy of $\pm 5 \%$. The relative humidity and the temperature are recorded at the beginning and the end of each measurement.

A radar beam scanning is performed with an angle of $\pm 10^{\circ}$ in azimuth and $\pm 10^{\circ}$ in elevation requiring the recording of 441 beat frequency spectra at different interrogation directions. Several estimators can be proposed in order to derive the relative humidity from the scattering data: (a) the value $\mathrm{e}_{\mathrm{Max}}$ of the unique - voxel containing the maximal echo level, (b) the mean echo level $e_{A}$ of all voxels values in the total interrogated volume, (c) the weighted mean echo level $e_{w}$ and (d) the shift $e_{\mu}$ of a log-normal model distribution of echo values (this estimator will be discussed further).

Measurements are first performed at a distance of $1.3 \mathrm{~m}$ between the radar and the sensor (near-field region). Estimators are computed on a volume from $1.2 \mathrm{~m}$ to $1.5 \mathrm{~m}$ comprising 3528 voxels. Calculating $\mathrm{e}_{\mu}$ requires the analysis of the echo level probability density function (PDF). Echo level values are fitted to a log-normal distribution, followed by a KolmogorovSmirnov test between the log-normal PDF and the measurement data. If the resulting two-tailed p-value of the test is lower than 0.90 , the echo level interval is reduced starting from the lowest values and ending to a success test with $\mathrm{p} \geq 0.90$. The log-normal model PDF is defined as follows:

$$
\phi(x)=\frac{K}{x \sigma \sqrt{2 \pi}} \cdot \exp \left\{\frac{-[\ln (\mu)-x]^{2}}{2 \sigma^{2}}\right\}
$$

where $\mathrm{K}$ is the scaling factor, $\mu$ denotes the shift (in volts) and $\sigma$ designates the standard deviation. The shift estimator $e_{\mu}$ is then given by $\exp (\mu)$. It has already been observed [6] that this estimator offers a large dynamic range. We define here a new very promising statistical estimator $\mathrm{e}_{\mathrm{x}}$ defined by the arithmetic mean of the four above-mentioned estimators, that is

$$
e_{X}=\frac{e_{A}+e_{W}+e_{\text {Max }}+e_{\mu}}{4} .
$$

As shown in Fig. 2, this estimator offers at $1.3 \mathrm{~m}$ a convenient linear variation with the relative humidity with a standard error of $0.009 \mathrm{~dB}$ and a large full-scale dynamic range of $12 \mathrm{~dB}$ from $38 \%$ RH to $69 \%$ RH. 


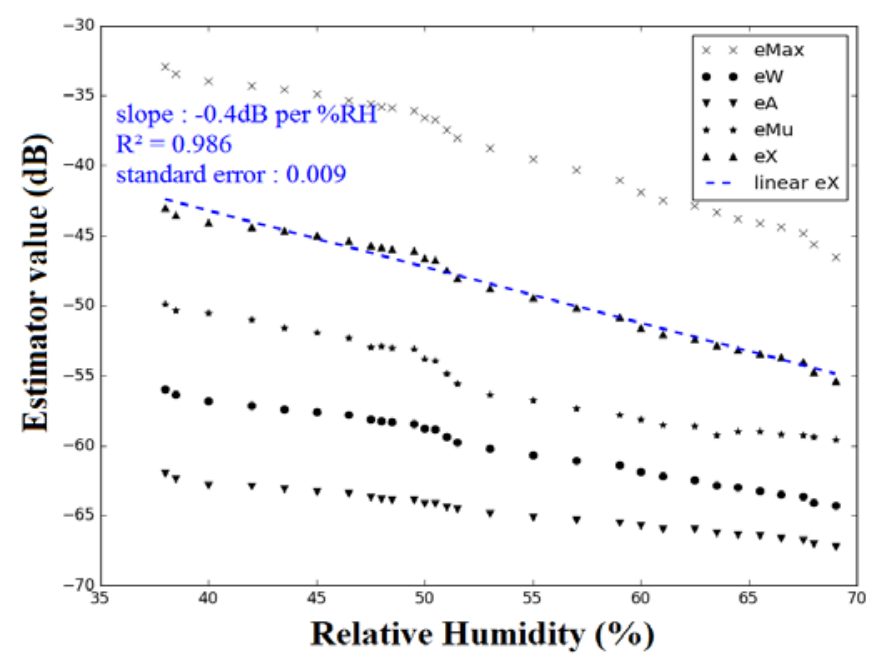

Fig. 2. Values of various estimators (see text for the corresponding definitions) vs. relative humidity from $38 \% \mathrm{RH}$ to $69 \% \mathrm{RH}$. The blue dashed curve is a linear regression of estimator $\mathrm{e}_{\mathrm{X}}$ computed values. The sensitivity of this estimator is found to be $-0.4 \mathrm{~dB}$ per $\mathrm{RH} \%$ with a standard error of $0.009 \mathrm{~dB}$.

B. Indoor interrogation of the humidity sensor placed at $10 \mathrm{~m}$ from the radar reader and linearity improvement of the humidity statistical estimator

It should be noted that a spherical beam scanning provides a spatial resolution at a distance $\mathrm{R}$ which depends on $R \times d \theta \times$ $d \varphi$. Consequently, as the interrogation distance increases, the voxels' size increases and the spatial resolution decreases. A solution to overcome this issue is to perform a linear translation of the radar during the measurement. The passive sensor is then interrogated at different incidence angles as done in the Synthetic Aperture Radar technique (SAR). This SAR approach has been used very recently with success in [7] for short range interrogation (few centimeters) of tags by using a MIMO radar.

To illustrate the method, the Van-Atta reflectarray is placed at 10.0 meters from the radar. The beam scanning method used in Section II. A is applied while a linear translation of the radar (perpendicular to the direction of interrogation) on a distance of $5 \mathrm{~cm}$ is performed with a step of $1 \mathrm{~cm}$. The resulting echo levels are converted into Cartesian coordinates to finally recombine the final SAR data grid. Estimators ex are shown on Fig. 3 for one single beam scanning (upper triangles) and for the SAR beam scanning (stars). The sensitivity remains the same at about $0.2 \mathrm{~dB}$ per \%RH. However, the standard error of the linear regression is significantly lower. This result justifies the linear translation of the radar for long interrogation distances in order to increase the measurement accuracy of the physical quantity (humidity here).

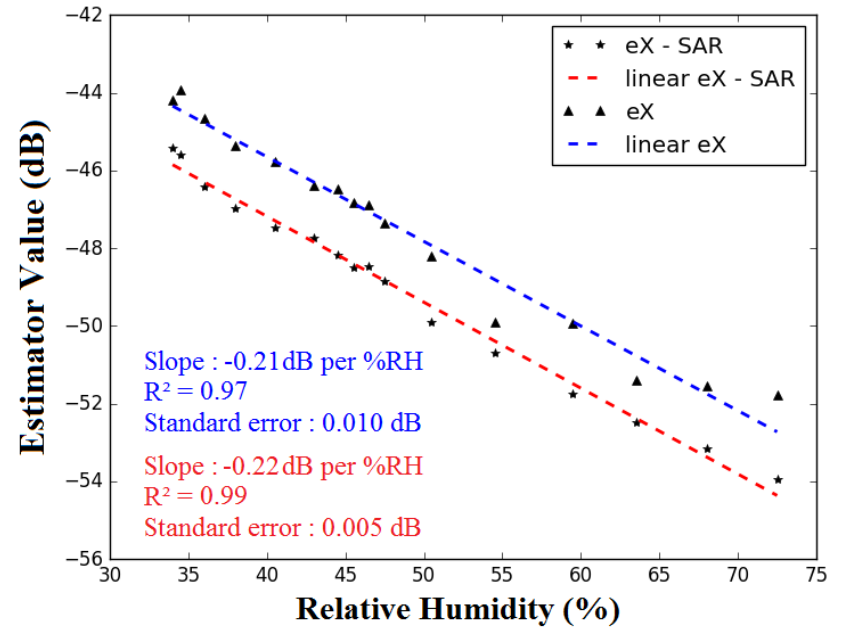

Fig. 3. Values of the estimator $\mathrm{e}_{\mathrm{x}}$ vs. relative humidity from $34 \% \mathrm{RH}$ to $74 \% \mathrm{RH}$ and for a humidity sensor placed at 10 meters from the radar. The standard error is reduced to half when translating the radar during the measurement compared with one obtained for a static radar.

\section{Long range radar interrogation (58 meters) of the passive humidity sensor and isosurfaces display}

The Van-Atta reflectarray is now located at 58.0 meters from the radar and the measured relative humidity is $48 \%$. A picture of the experimental setup is shown in Fig.1(b). Because of the fixed number of 1024 samples of the used chirp up-ramp frequency modulation, it was no more possible for this distance to perform a measurement with a bandwidth B of $2 \mathrm{GHz}$. The bandwidth can be set to $1.2 \mathrm{GHz}$ leading to a lower theoretical depth resolution $(\mathrm{d}=12,5 \mathrm{~cm})$. Moreover, the $\mathrm{x}$-range and $\mathrm{y}$ range resolutions are found to be equal to $1.0 \mathrm{~m}$ for a $1^{\circ}$ mechanical step.

Fig. 4 is the three-dimensional (3D) representation of the ambient echo (clutter) level at distances (z-direction) between 57.5 meters and 58.5 meters. It is displayed with isosurfaces (layers of same echo level) which are overimposed on the 3D Cartesian grid. The estimator values for the passive sensor echo levels over the isosurfaces are $\mathrm{e}_{\mathrm{X}}=-64.2 \mathrm{~dB}$ and $\mathrm{e}_{\mathrm{Max}}=-56.2 \mathrm{~dB}$. The Van-Atta reflectarray is visible among the clutter with a dynamic range difference of around $6 \mathrm{~dB}$, knowing that the maximal echo from the clutter is $e_{\text {Max }}=-62.1 \mathrm{~dB}$. Despite a lower depth and cross-range resolutions, it is very encouraging to observe that the echo level of the clutter is very small compared to the humidity sensor echo at such long distances. The maximal echo level value should be $5 \mathrm{~dB}$ higher for relative humidity values around $35 \%$. Consequently, the full-scale dynamic range is around $11 \mathrm{~dB}$. This kind of long range indoor interrogation is possible by using the cross-polarization effect of the reflectarray: the depolarization becomes a discriminant criteria because the environment does not depolarize as much as the passive sensor.

Same measurements are then analyzed but only with specific angles of incidence in azimuth. Fig. 5 shows the value of the estimator $\mathrm{e}_{\mathrm{x}}$ in function of the angle of incidence at a distance 
of 58.0 meters. A decrease of $2 \mathrm{~dB}$ is observed at an angle of $\pm 2^{\circ}$ around the normal incidence, and a decrease of $4 \mathrm{~dB}$ to $8 \mathrm{~dB}$ at an angle of $\pm 5^{\circ}$. These results are explained by: (a) a directive backscattering of the Van-Atta reflectarray and (b) distance inaccuracies at long range of interrogation. Indeed, an angular resolution of $1^{\circ}$ at $58.0 \mathrm{~m}$ generates a tangential projection of $1.0 \mathrm{~m}$. To overcome this backscattering degradation, it is preferable to perform measurements with several angles of interrogation and thus to ensure the remote reading with a normal incidence.

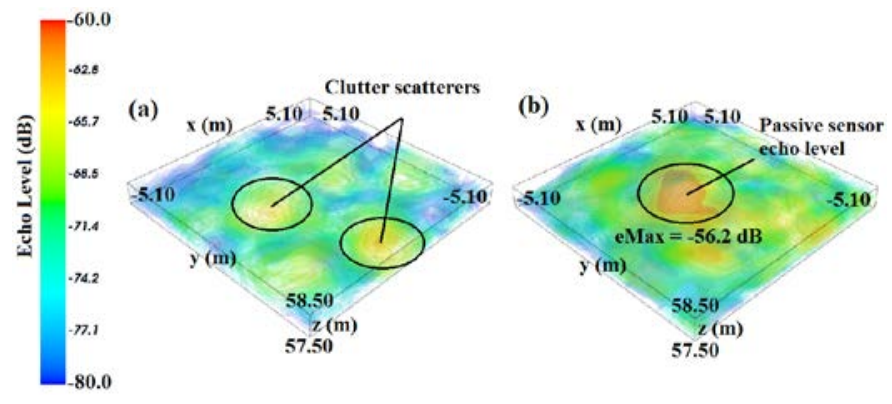

Fig. 4. Isosurfaces display of echo levels for an indoor measurement at 58.0 meters: (a) in absence of the passive humidity sensor and (b) in presence of the passive humidity sensor $(\mathrm{RH}=48 \%)$.

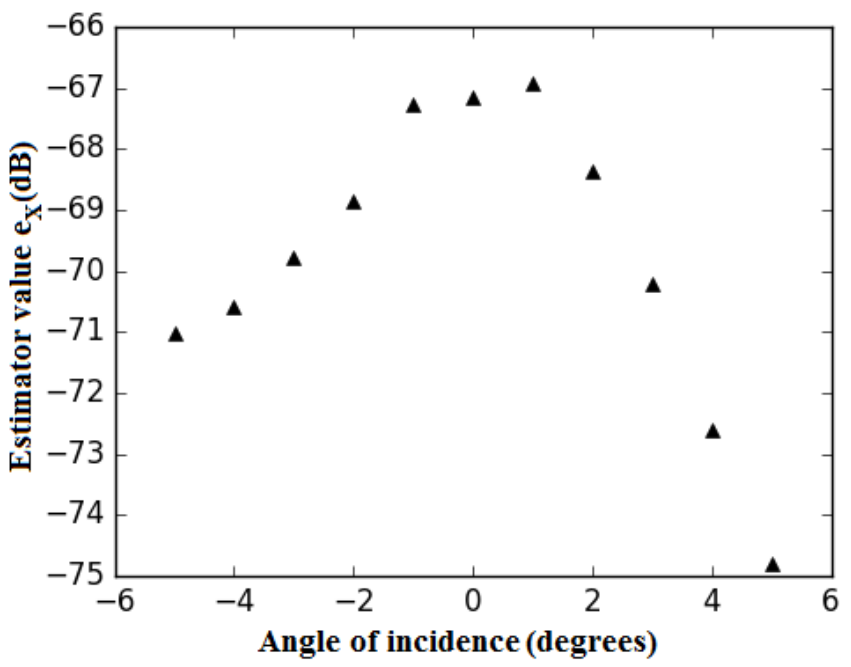

Fig. 5. Value of the estimator $\mathrm{e}_{\mathrm{X}}$ vs angle of incidence for a Van-Atta reflectarray interrogated at 58 meters.

\section{CONCLUSION}

This paper has demonstrated the feasibility of wireless interrogation of passive sensors at distances up to 58 meters. This was possible thanks to the use of a Van-Atta reflectarray as well as a Synthetic Aperture Radar technique and 3D radar imagery. A measurement sensitivity of $0.2 \mathrm{~dB}$ to $0.4 \mathrm{~dB}$ per $\% \mathrm{RH}$ was measured as a linear variation of the proposed estimator with a standard error of $\pm 0.005 \mathrm{~dB}$. Long-range indoor measurements demonstrate an estimated full-scale dynamic range over $11 \mathrm{~dB}$ over ambient clutter. This performance can be reached with a normal angle of incidence.

\section{ACKNOWLEDGMENT}

This work was partially funded by the French Technological Research Agency (Agence Nationale de la Recherche Technologique, France) and Ovalie-Innovation

\section{REFERENCES}

[1] D. Henry, H. Aubert and P. Pons, "3D scanning and sensing technique for the detection and remote reading of a passive temperature sensor," 2016 IEEE MTT-S International Microwave Symposium (IMS), San Francisco, CA, 2016, pp. 1-4.

[2] J. G. D. Hester and M. M. Tentzeris, "Inkjet-printed Van-Atta reflectarray sensors: A new paradigm for long-range chipless low cost ubiquitous Smart Skin sensors of the Internet of Things," 2016 IEEE MTT-S International Microwave Symposium (IMS), San Francisco, CA, 2016, pp. 14

[3] J. G. D. Hester; M. M. Tentzeris, "Inkjet-Printed Flexible mm-Wave VanAtta Reflectarrays: A Solution for Ultralong-Range Dense Multitag and Multisensing Chipless RFID Implementations for IoT Smart Skins," in IEEE Transactions on Microwave Theory and Techniques , vol.PP, no.99, pp.1-11

[4] S.O. Piper, "Receiver frequency resolution for range resolution in homodyne FMCW radar," Telesystems Conference: Commercial Applications and Dual-Use Technology, 16-17 June 1993, pp.169-173

[5] E. M. Amin, N. C. Karmakar, and B. Winther-Jensen, "Polyvinyl-alcohol (pva)-based RF humidity sensor in microwave frequency," Progress In Electromagnetics Research B, Vol. 54, 149-166, 2013.

[6] D. Henry, H. Aubert and P. Pons, "Wireless passive sensors interrogation technique based on a three-dimensional analysis," 2016 46th European Microwave Conference (EuMC), London, 2016, pp. 49-52.

[7] M. Zomorrodi and N. C. Karmakar, "Novel MIMO-based technique for EM-imaging of chipless RFID," 2015 IEEE MTT-S International Microwave Symposium, Phoenix, AZ, 2015, pp. 1-4 